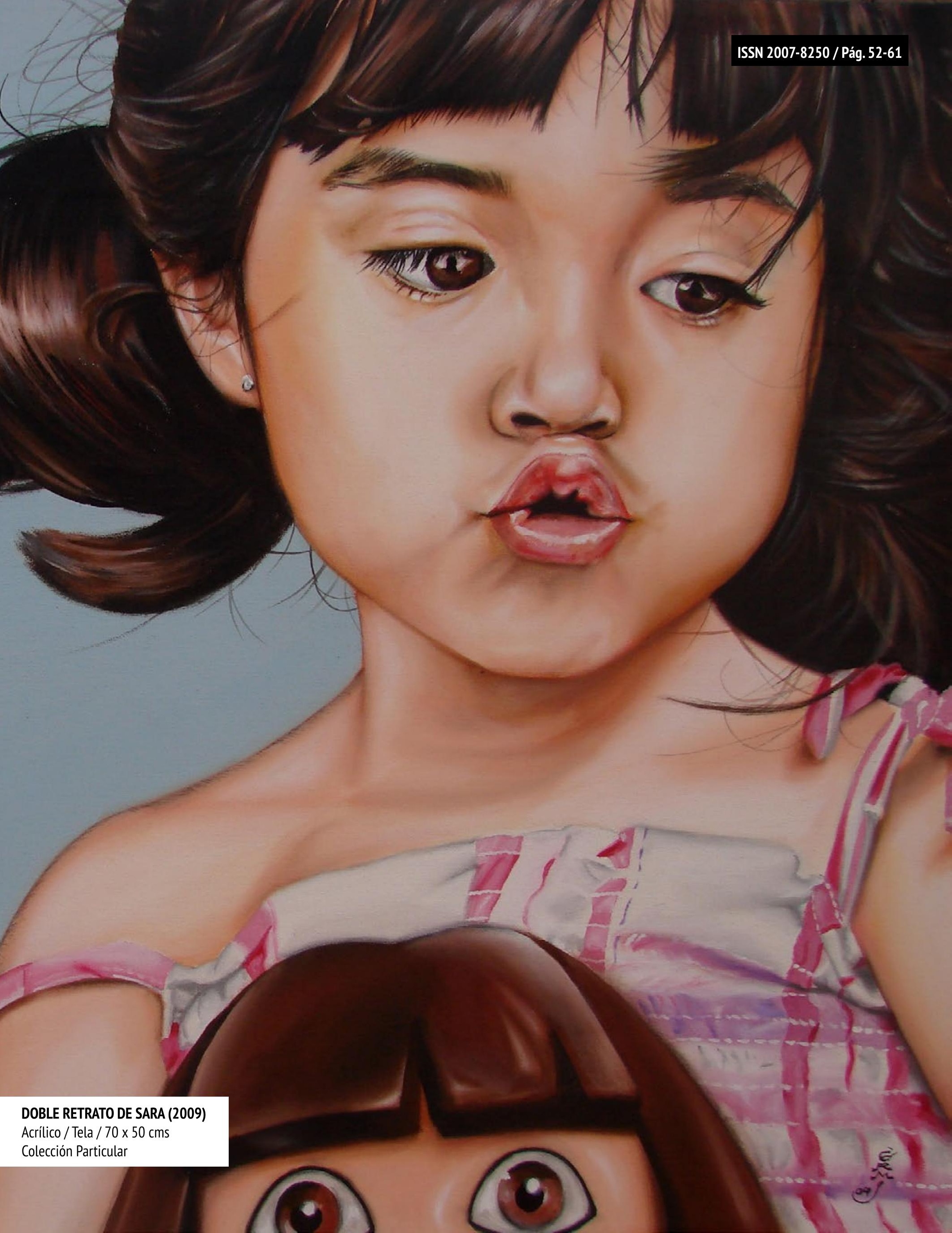




\title{
https://doi.org/10.29105/pu8.16-5 \\ ELABORACIÓN DE RECURSOS EDUCATIVOS DIGITALES \\ EN CONTEXTOS UNIVERSITARIOS
}

\section{PLANNING OF ADVANCED INSTRUCTIVE ASSETS IN COLLEGE SETTINGS}

\author{
LETICIA PADILLA FACUNDO \\ https://orcid.org/0000-0002-6492-4013 \\ ANA IRENE CUEVAS GUTIÉRREZ \\ https://orcid.org/0000-0002-3019-4745
}

\section{RESUMEN}

El presente proyecto busca generar una guía que apoye al docente en la elaboración de recursos educativos digitales pertinentes para los contextos universitarios actuales, esto debido a la verdadera necesidad que tienen las Universidades e instituciones de Educación Superior de avanzar hacia una verdadera transformación digital, que implica entre otras cosas, contar con materiales didácticos adecuados que permitan ofrecer una verdadera educación digital de calidad. No se busca que el docente tenga conocimiento de los recursos digitales, sino que, a partir de los contenidos de sus propias unidades de aprendizaje, sepa desarrollar las estrategias de aprendizaje adecuadas a las competencias que espera alcanzar, utilizando los recursos electrónicos digitales (RED) como medio, no como el modo para lograrlo.

PALABRAS CLAVE:

Guía, Didáctico, Calidad, Digital, Recurso, Educación
ABSTRACT

This project seeks to generate a guide that supports the teacher in the development of relevant digital educational resources for current university contexts, this due to the true need that Universities and Higher Education institutions have to move towards a true digital transformation, which implies among other things, having adequate teaching materials that allow us to offer a true quality digital education. It is not intended that the teacher has knowledge of digital resources, but that, based on the contents of their own learning units, they know how to develop the appropriate learning strategies for the skills they hope to achieve, using digital electronic resources (RED) as a means, not as the way to achieve it.

KEYWORDS: Guide, Didactic, Quality, Digital, Resource, Education 
L

os tiempos actuales nos hacen un llamado para entender las Tecnologías de la Información y la Comunicación (TIC), y cómo éstas han tomado un papel cada vez más relevante en el sector educativo, el cual se ha visto obligado a transformarse con cambios cada vez más vertiginosos, generando nuevas formas de enseñanza y aprendizaje que parten de la integración de prácticas educativas innovadoras.

Para Hernández (2017) este uso de las TIC ha impulsado un nuevo ambiente de aprendizaje donde el estudiante debe ser capaz de convertirse en el protagonista de su propio aprendizaje, gracias al tiempo y la flexibilidad que estas herramientas proporcionan. Además dentro de esta transformación se espera que los docentes, en su papel de facilitadores, sean los encargados no sólo de ofrecer, sino también de generar recursos educativos de calidad que puedan impulsar el aprendizaje de los estudiantes ante estos nuevos contextos digitales.

El fácil acceso a una educación de calidad es uno de los grandes retos que presenta la educación actual. Ante

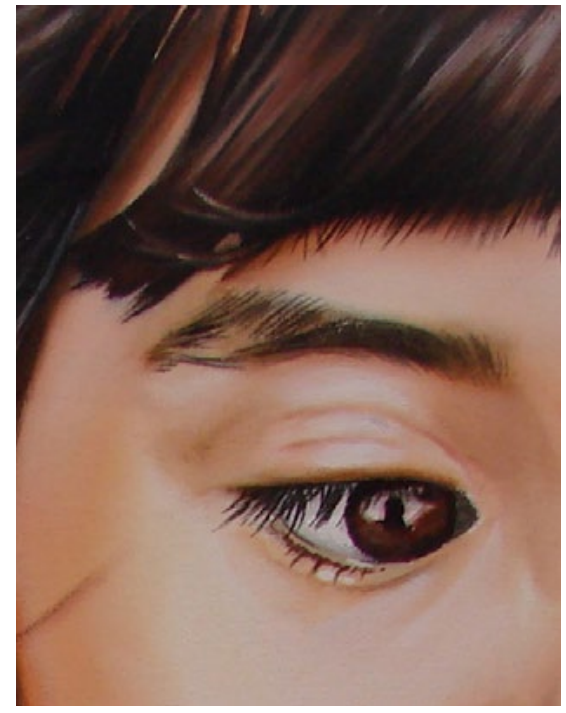

las nuevas necesidades que impulsan modelos educativos a distancia, la implementación de recursos educativos digitales (RED) permitirán desarrollar una autonomía educativa en el estudiante, ya que el profesor puede centrarse en áreas de conocimiento más significativas y específicas.

Hablar de un aprendizaje exitoso en esta modalidad debe de considerar distintos aspectos, entre ellos el de ofrecer materiales educativos que permitan que una persona pueda aprender de forma exitosa sin necesidad de estar físicamente en una institución. El presente proyecto busca responder

\section{Una característica importante de los materiales} de instrucción que se utilizan en la educación virtual es que deben contar con una estructura pedagógica bien definida para que cumplan con su finalidad de reforzar los aprendizajes. 
educativos digitales pueden facilitar la comprensión, la interpretación y la apropiación de la información; sin embargo, por sí solos no garantizan efectividad en el logro de aprendizajes significativos.

En los últimos años, el mundo ha sido testigo de diversos cambios debido a la llegada de las Tecnologías de la Información y la Comunicación, las cuales se han convertido en un aspecto esencial en cualquier ámbito de nuestras vidas a tal punto que hoy resulta imposible imaginarnos sin ellas. Las TIC son todos aquellos recursos, herramientas y programas que se utilizan para procesar, administrar y compartir la información mediante diversos soportes tecnológicos, tales como: computadoras, teléfonos móviles, televisores, reproductores portátiles de audio y video o consolas de juego.

Las TIC han revolucionado nuestras vidas y han ido ocupando un papel fundamental debido a que nos permiten estar en comunicación y acceder a una gran cantidad de información, gracias a las redes informáticas como el Internet. Con él, la medicina, el comercio, la comunicación, así como cualquier otro

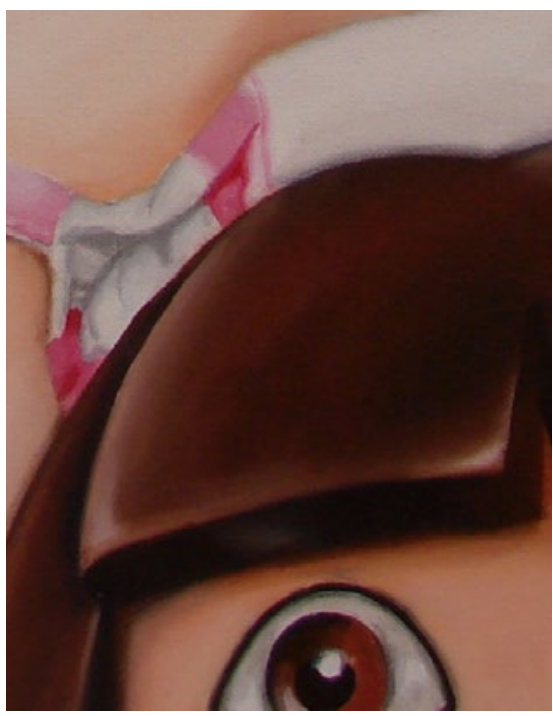

Aguilar, De la Vega, Lugo y Zarco (2014) afirman que los materiales educativos son uno de los elementos del sistema educativo que han evolucionado notoriamente gracias a las nuevas herramientas informáticas, siendo más diversos y complejos.

tipo de trabajo 0 actividad se hace de forma distinta y por supuesto, sería impensable que nos mantuviéramos inmóviles respecto a la educación (García, 2020, p. 26). Es por ello por lo que hoy en día resulta difícil pensar en la educación sin ligarla de forma estrecha con las TIC, especialmente tratándose de la educación superior, esto debido a las grandes bondades que la tecnología ofrece para facilitar y mejorar el proceso educativo.

\section{RECURSOS \\ EDUCATIVOS DIGITALES}

Gracias a la llegada de las Tecnologías de la Información y la Comunicación y

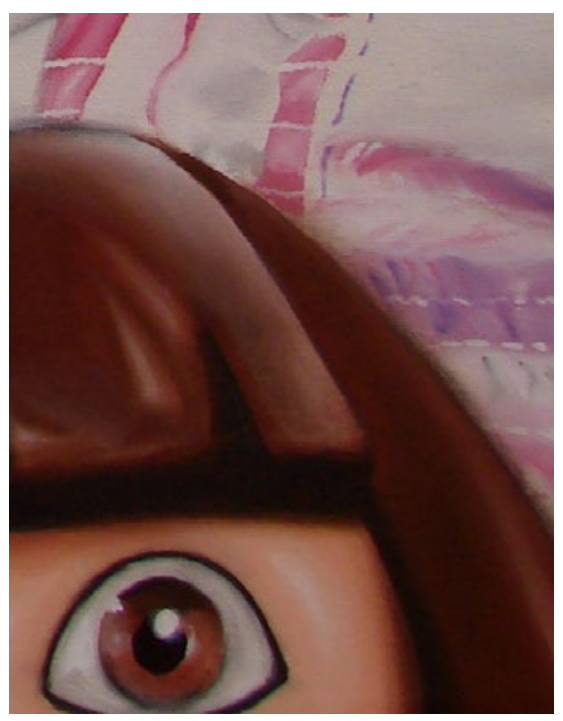

con ello, la era digital, ha sido posible la generación de una gran cantidad de materiales educativos con fines didácticos que, por su formato y flexibilidad están disponibles en todo momento para apoyar en el proceso enseñanza-aprendizaje.

Al respecto, Aguilar, De la Vega, Lugo y Zarco (2014) afirman que los materiales educativos son uno de los elementos del sistema educativo que han evolucionado notoriamente gracias a las nuevas herramientas informáticas, siendo más diversos y complejos.

A raíz de la educación digital, ha sido fundamental el desarrollo de materiales didácticos precisamente digitales, que estén a la par con esta nueva modalidad de enseñanza. Es por ello que ha surgido una nueva categoría en la clasificación de los materiales didácticos, los CODIE (Contenido Digital Educativo), estos conservan las propiedades de un material educativo tradicional pero además obtienen nuevas características gracias al formato digital que les da propiedades de reusabilidad en cualquier lugar y momento (Aguilar, De la Vega, Lugo y Zarco, 2014). 


\section{El profesor no debe desentenderse de su tarea}

\section{que consiste en guiar los procesos de búsqueda,}

analizar y seleccionar la información, sintetizar

el contenido y difundirlo. (Viñals y Cuenca, 2016)

Los materiales didácticos utilizados en la enseñanza digital cuentan con una amplia variedad debido a que se encuentran en distintas presentaciones siempre y cuando se encuentre en formato digital, lo que permite que sea utilizado con fines educativos. De acuerdo con Aguilar, De la Vega, Lugo y Zarco (2014) a este tipo de materiales se le puede dividir en tres subgrupos diferenciados por su nivel de agrupación y complejidad: los recursos digitales, el software educativo y los entornos virtuales de aprendizaje, siendo los Recursos Educativos Digitales los de nuestro interés para el desarrollo de este trabajo.

\section{DISEÑO INSTRUCCIONAL DE UN RED}

La era digital ha permitido acceder a una mayor cantidad de información, así como ampliar y diversificar la interacción entre docentes y estudiantes, gracias a la utilización eficaz de la tecnología, lo cual sólo resulta posible si el docente asume su papel de facilitador y desarrolla ambientes de aprendizaje idóneos, mediante el diseño y elaboración de materiales altamente significativos como los Recursos Educativos Digitales, que con un diseño y

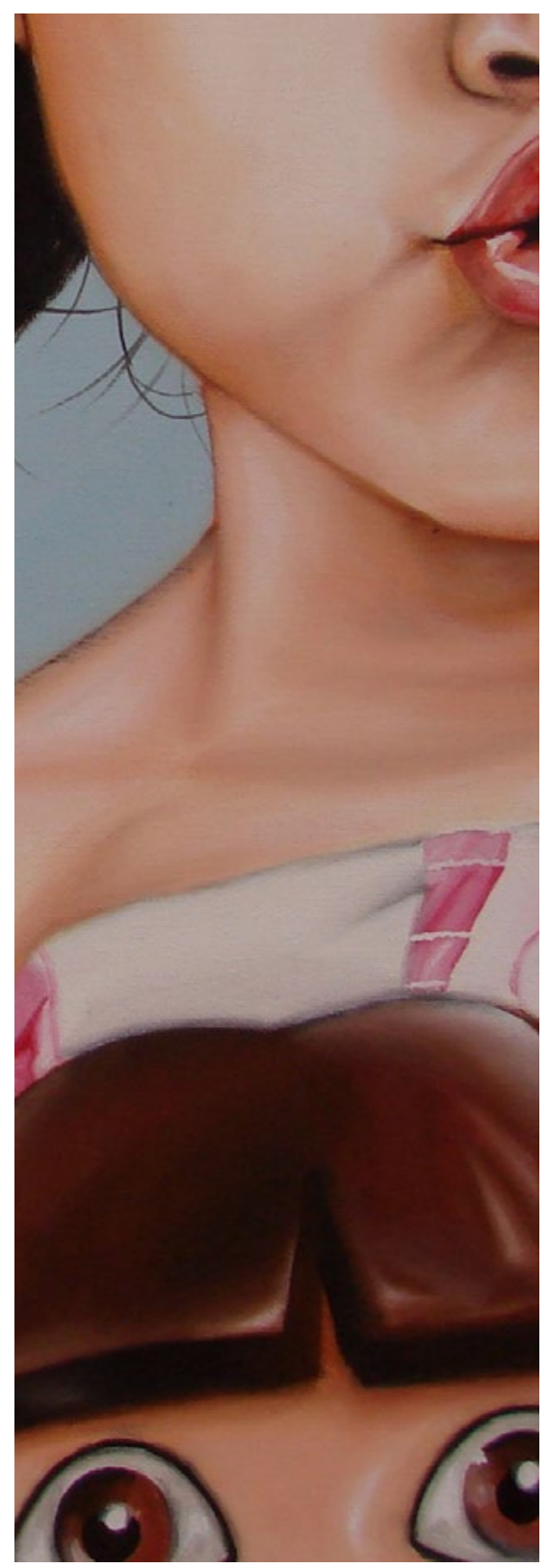

Vinueza y Simbaña (2017) afirman:

Las TIC cuentan con un amplio campo de aplicación, y generan muchas ventajas siempre y cuando sean aplicadas correctamente, la educación no podía ser la excepción y es allí donde el rol del docente es percibir todos los ámbitos de esta rama tecnológica de lo contrario no podrían hacer que sus alumnos y por ende la universidad donde trabajan accedan a una educación de calidad (p.365).

El docente es un facilitador y como tal, debe dar autonomía al estudiante, sin embargo, en la era digital el conocimiento está en la red y es abundante, por lo que el profesor no debe desentenderse de su tarea que consiste en guiar los procesos de búsqueda, analizar y seleccionar la información, sintetizar el contenido y difundirlo (Viñals y Cuenca, 2016). Es ahí donde se convierte en un diseñador de escenarios óptimos de acuerdo con las necesidades de sus estudiantes.

Al realizar un diseño instruccional, el profesor se ocupa de la planeación, la preparación y el diseño de los recursos y ambientes necesarios para que se lleve a cabo el aprendizaje. Por ello 
en el caso de los RED, el profesor debe realizar un diseño previo para que estos cumplan con las características necesarias para ser considerados de calidad y sean los apropiados para el entorno donde serán presentados.

Al respecto, Viñals y Cuenca (2016) afirman lo siguiente:

El rol de experto en instrucción consiste en que el docente aporte todo el conocimiento, imaginación y creatividad posible para hacer el proceso de aprendizaje del alumno efectivo y atractivo. Para lograrlo el experto debe convertirse en un auténtico diseñador de originales experiencias de aprendizaje y, a su vez, debe practicar el arte de realizar preguntas adecuadas que inciten a que los alumnos reflexionen y reconsideren un punto de vista (p.111).

En el caso de los RED, como se ha mencionado, ofrecen distintas oportunidades al impulsar el proceso de enseñanza aprendizaje sin embargo requieren de un diseño previo que los convierta en óptimos para cumplir su función. García (2006), afirma que los recursos digitales están revolucionando las prácticas educativas, pero resulta necesario que el docente sepa identificar determinados factores en ellos y no sean usados en el aula sin haber cumplido con los mínimos requisitos en cuanto a su calidad. Es decir, que deben contar con un trabajo previo para que sean claros y efectivos, que y ayuden al alumno a desarrollar las distintas capacidades.

Por lo anterior, se propone la elaboración de una guía docente que permita contribuir a la generación de una educación de calidad en escenarios

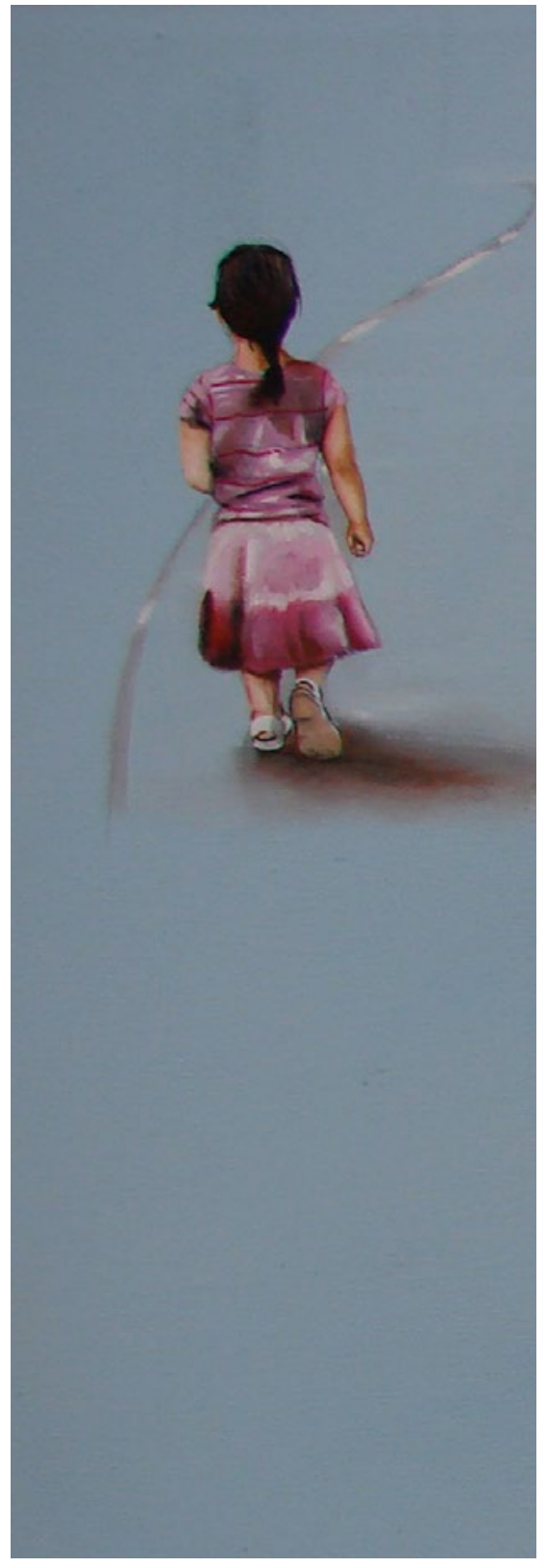

virtuales, por medio de la elaboración de recursos educativos digitales que reúnan las características necesarias para ser aplicados en un contexto universitario.

El objetivo es la elaboración de una guía docente que a su vez le permita la realización de recursos educativos digitales en contextos universitarios.

A partir de lo siguiente:

- Definir qué se considera un recurso educativo digital y reconocer su importancia en la actualidad para la enseñanza universitaria.

- Identificar los aspectos necesarios para el diseño de recursos educativos digitales a fin de que éstos sean apropiados para el aprendizaje de los estudiantes sin necesidad de estar presencialmente en una institución.

\section{COMPETENCIAS}

En el caso de la Educación Superior, las tecnologías de la información y comunicación ofrecen nuevos entornos que permiten el desarrollo de competencias necesarias para el aprendizaje y generadoras de habilidades para la vida (García, Reyes y Godínez, 2017).
García (2006), afirma que los recursos digitales están revolucionando las prácticas educativas, pero resulta necesario que el docente sepa identificar determinados factores en ellos y no sean usados en el aula sin haber cumplido con los mínimos requisitos en cuanto a su calidad. 


\section{Calderón y Loja (2017) señalan que el docente}

\section{del siglo XXI está Ilamado a la aplicación}

\section{de distintas herramientas tecnológicas,}

\section{para ello resulta necesario que el profesor cuente}

\section{con los distintos conocimientos que le permitan}

proveer materiales didácticos apropiados

\section{para ser utilizados en la educación digital.}

Éstas permiten que los futuros profesionistas cuenten con las herramientas necesarias para enfrentar los retos actuales que requieren de innovación y solución de distintas problemáticas. Sin embargo, para acceder a estos múltiples beneficios que las TIC ofrecen, se requiere trabajar para ofrecerlas a los estudiantes en una presentación adecuada para su aprendizaje

\section{COMPETENCIAS A DESARROLLAR}

La labor docente en la era digital tiene que ver con la planificación, la intervención, la comunicación o la propia actualización; y en todos esos ámbitos deberá ser competente en el uso de la tecnología. A raíz del uso de esta guía se espera que los docentes puedan ser competentes digitales al adoptar estas dos dimensiones propuestas por Viñals y Cuenca (2016):

1. Información. Identificar, localizar, recuperar, almacenar, organizar y analizar la información digital, evaluando su finalidad y la relevancia.

2. Creación de contenido. Crear y editar contenidos nuevos (textos, imágenes, videos, entre otros), integrar y reelaborar conocimientos y contenidos previos, realizar producciones artísticas, contenidos multimedia y programación informática, saber aplicar los derechos de propiedad intelectual y las licencias de uso.

\section{CONTENIDOS}

Este proyecto está encaminado a apoyar al profesor universitario, el cual debe de evolucionar y adaptarse a esta nueva forma de ver la educación, la cual está marcada por la era digital. Calderón y Loja (2017) señalan que el docente del siglo XXI está llamado a la aplicación de distintas herramientas tecnológicas, para ello resulta necesario que el profesor cuente con

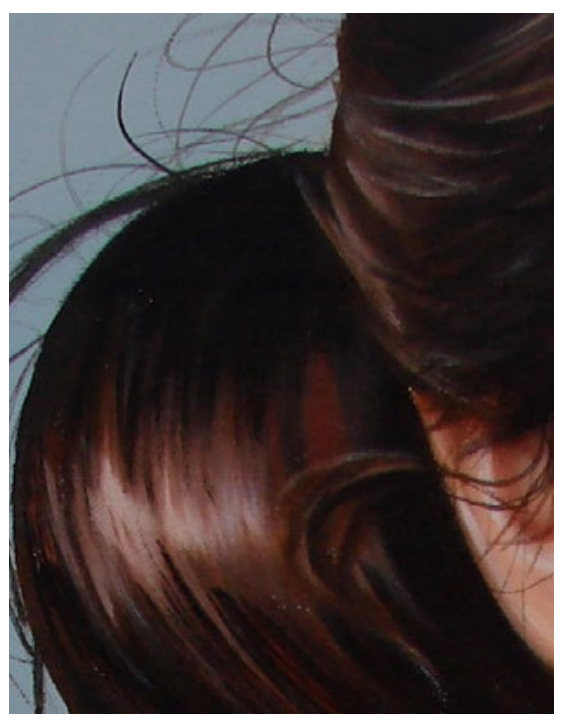

los distintos conocimientos que le permitan proveer materiales didácticos apropiados para ser utilizados en la educación digital. En esta guía se busca reunir los aportes de distintos autores que serán de base para que los docentes universitarios cuenten con las habilidades y conocimientos necesarios para desarrollar los recursos educativos digitales.

\section{METODOLOGÍA}

Proyectos como éste, buscan que el docente pueda reflexionar sobre la importancia que tienen las TIC para la educación actual, partiendo del conocimiento de que la tecnología por sí sola no tiene un impacto en el proceso formativo, sino que requiere de un previo diseño y educación por parte del docente, para que estas herramientas puedan contribuir al proceso de aprendizaje .

Viñals y Cuenca (2016, p. 112) señalan lo siguiente:

La tecnología y la información por sí solas no guían, no ayudan y ni aconsejan al alumnado; por ello, la labor del docente en la educación digital es hoy más importante que nunca.

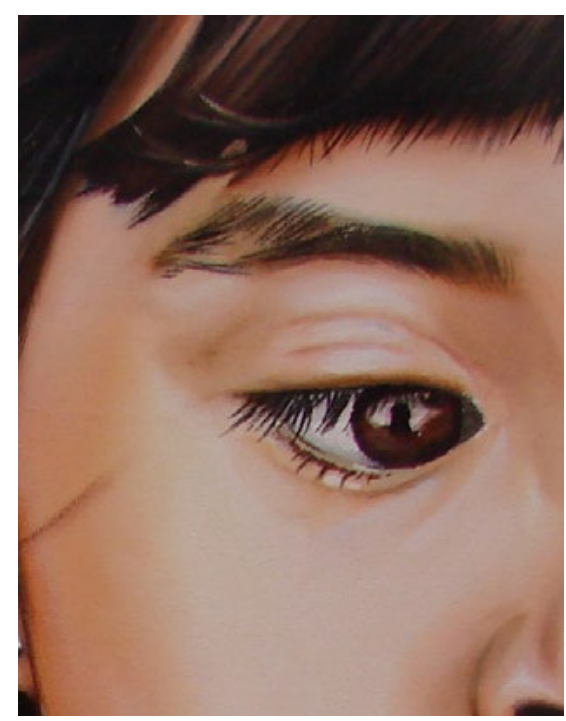




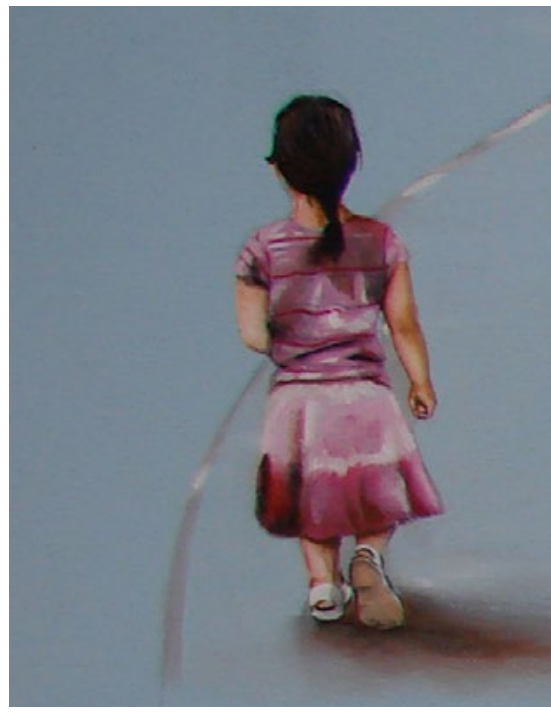

Una guía para la elaboración de recursos educativos digitales en contextos universitarios, tiene el objetivo de que el docente sepa identificar los aspectos necesarios para diseñar y elaborar recursos educativos de calidad y apropiados para el aprendizaje de los estudiantes universitarios. Pero sin duda, el beneficio principal será el impacto que traerá en el aprendizaje de los estudiantes, quienes estarán recibiendo una educación de calidad de acuerdo a los requerimientos de la era digital.

\section{CONOCIMIENTOS PREVIOS}

El profesor debe de tener conocimientos sobre la docencia y el uso de estrategias de aprendizaje, así como un conocimiento básico sobre aspectos relacionados con las tecnologías de la información y la comunicación.

\section{AMBIENTE DE APRENDIZAJE}

Gargallo et al. (2017) señalan que el profesor es el mediador entre el contenido y el alumno, siendo su tarea fundamental la de articular buenos ambientes y experiencias de aprendizaje mediante el uso de diversos métodos, procurando utilizar una metodología innovadora, que permitan al alumnado el adquirir los resultados por docentes de la Universidad Autónoma de Nuevo León, pero en general por profesores universitarios, permitiendo obtener los conocimientos necesarios para poder elaborar recursos educativos digitales en sus diferentes contextos educativos.

No se trata sólo de que los docentes reconozcan los recursos educativos digitales, sino que haya una verdadera sensibilización para saber cómo aplicarlos apropiadamente de acuerdo a las necesidades. Ésta será la base para un correcto diseño instruccional, ya que no se busca que el docente tenga conocimiento de los recursos digitales, sino que, a partir de los contenidos de sus propias unidades de aprendizaje, sepa desarrollar las estrategias de aprendizaje adecuadas a las competencias que espera alcanzar, utilizando los RED como medio, no como el modo para lograrlo.

Finalmente se espera que, con la implementación de esta guía, no sólo obtenga un aporte positivo para el quehacer docente, sino que también sea de beneficio para el aprendizaje de los estudiantes, quienes serán los beneficiarios indirectos de esta propuesta.

No se trata sólo de que los docentes reconozcan los recursos educativos digitales, sino que haya una verdadera sensibilización para saber cómo aplicarlos apropiadamente de acuerdo

a las necesidades. 


\section{REFERENCIAS}

Aguilar I., De la Vega J., Lugo, 0. y Zarco, A. (2014). Análisis de criterios de evaluación para la calidad de los materiales didácticos digitales. Revista Iberoamericana de Ciencia, Tecnología y Sociedad-CTS, 9 (25), enero-, 2014,73-89. Disponible en: http://www.redalyc.org/articulo.oa?id=92429919005

Calderón, M. y Loja, H. (2018). Un cambio imprescindible: el rol del docente en el siglo XXI. ILLARI, (6), 35-40. Disponible en: https://www.aacademica.org/ margarita.calderon/2

García, B, A. (2016). Evaluación de recursos tecnológicos didácticos mediante e-rúbricas. RED. Revista de Educación a Distancia, (49), 1-13. Disponible en: http://www.redalyc.org/articulo.oa?id=54745362013

García, L. (2020). Bosque semántico: ¿educación/enseñanza/aprendizaje a distancia, virtual, en línea, digital, eLearning? RIED. Revista Iberoamericana de Educación a Distancia, 23 (1), pp. 09-28. Disponible en: http://dx.doi.org/10.5944/ ried.23.1.25495

García, M., Reyes, J. y Godínez, G. (2017). Las Tic en la educación superior, innovaciones y retos. Revista Iberoamericana de las Ciencias Sociales y Humanisticas, 6(12). Recuperado de: http://ricsh.org.mx/index.php/RICSH/article/ view/135/727

Gargallo, B., Pérez C., Jiménez, M., Martínez, N., y Giménez, J. (2017). Métodos centrados en el aprendizaje, implicación del alumno y percepción del contexto de aprendizaje en estudiantes universitarios. Educación XX1, 20(2),161-187. ISSN: 1139-613X. En: https://www.redalyc.org/articulo.0a?id=70651145007

Hernández, R. M. (2017). Impacto de las TIC en la educación: Retos y Perspectivas. propósitos y Representaciones, 5(1), 325 - 347. Disponible en: http://dx.doi. org/10.20511/pyr2017.v5n1.149

Pérez, 0, I. (2017). Creación de recursos educativos digitales: Reflexiones sobre la innovación educativa con TIC. RISE - International Journal of Sociology of Education, 6(2), 244-268. Recuperado de: https://www.redalyc.org/ pdf/3171/317151451004.pdf

Vinueza V.S.F. y Simbaña G.V. P. (2017). Impacto de las TIC en la Educación Superior en el Ecuador. Revista Publicando, 4 No 11. (1). 2017, 355-368. ISSN 1390-9304.

Viñals, A. y Cuenca, J. (2016). El rol del docente en la era digital. Revista Interuniversitaria de formación del Profesorado, 30(2), 103-114. Disponible en: http:// www.redalyc.org/articulo.oa?id $=27447325008$

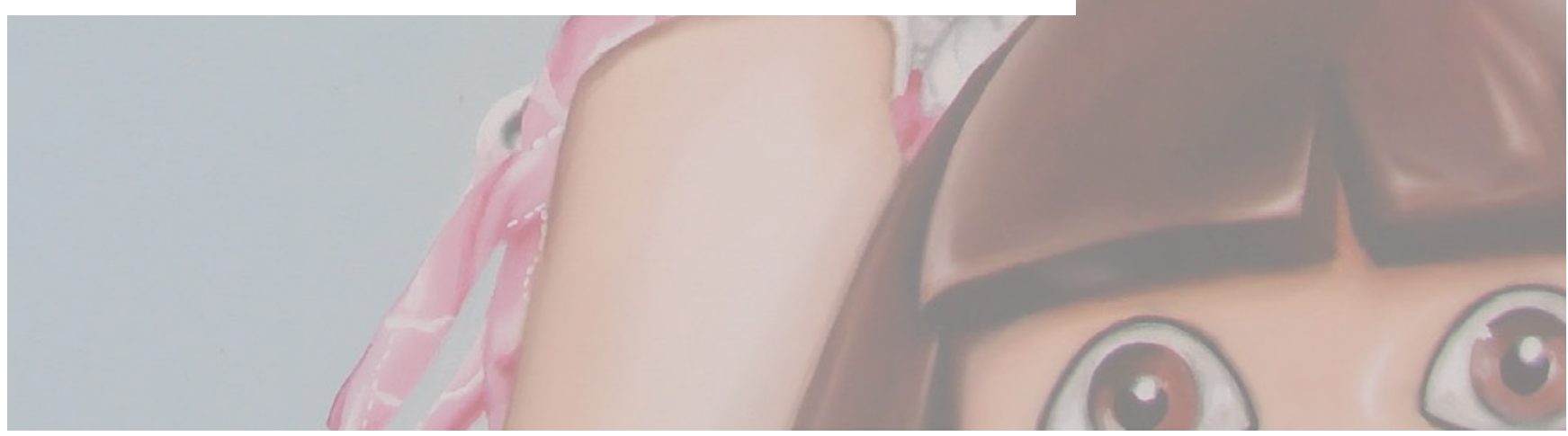




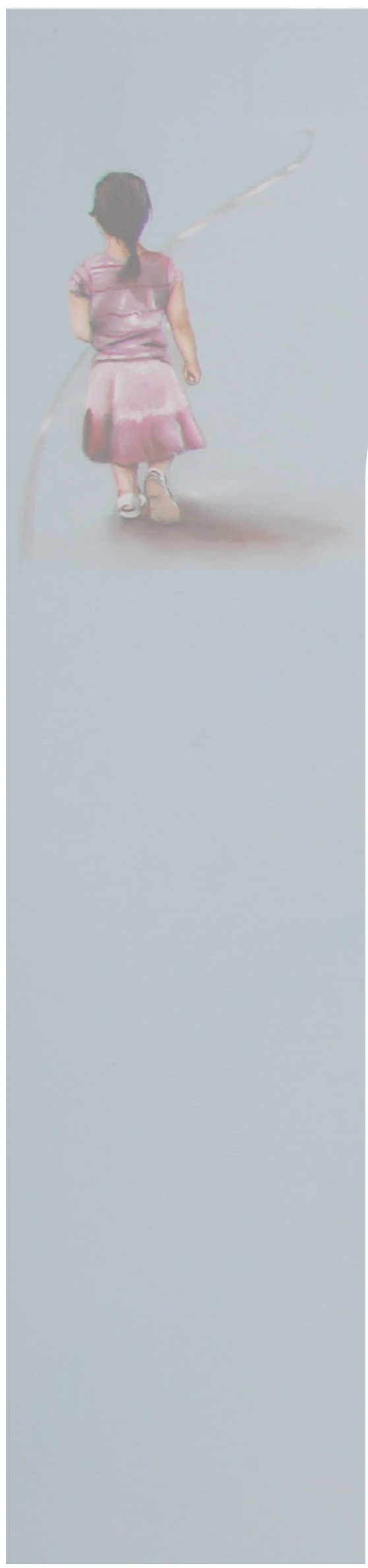

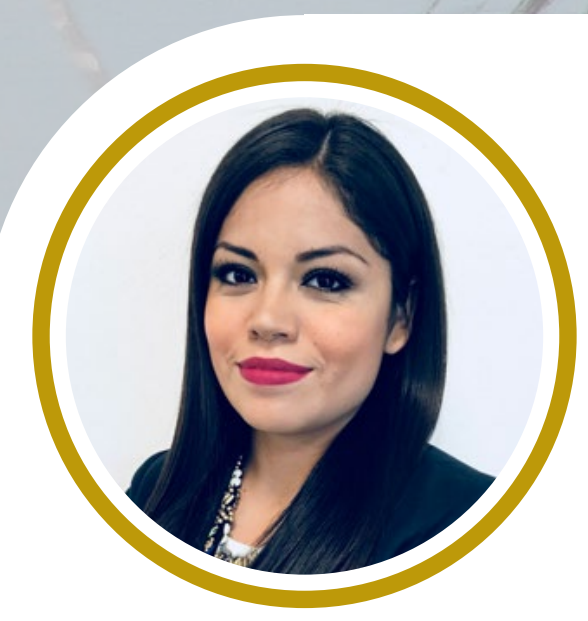

LETICIA PADILLA FACUNDO

Licenciada en Ciencias del Lenguaje con acentuación en enseñanza del inglés por la Facultad de Filosofía y Letras de la Universidad Autónoma de Nuevo León. Cuenta con estudios de Maestría en Docencia con Orientación en Educación Media Superior en la Facultad de Psicología de la UANL. Se ha desempeñado como docente en la preparatoria 22 de la UANL. Actualmente trabaja en la Secretaría de Innovación y Desarrollo Digital de la UANL donde participa en distintas producciones audiovisuales desarrolladas por la Dirección de Radio y Televisión Universitaria.

Correo Electrónico: letticia.padilla@gmail.com

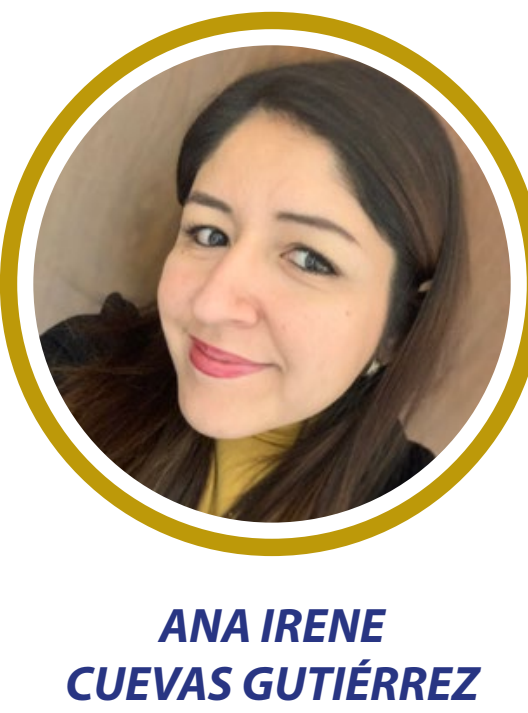

Doctora en Filosofía con acentuación en Psicología por la Universidad Autónoma de Nuevo León. Docente de la Facultad de Filosofía y Letras y de la Facultad de Psicología. Miembro de la Red de Investigación Educativa, UANL y miembro del Comité de la Maestría en Docencia del Nivel Medio Superior. Es autora de diversos artículos y capítulos de libros. Actualmente trabaja en la Secretaría Académica de la Universidad Autónoma de Nuevo León en el área de Investigación Educativa.

Correo Electrónico: ania.ny@gmail.com 


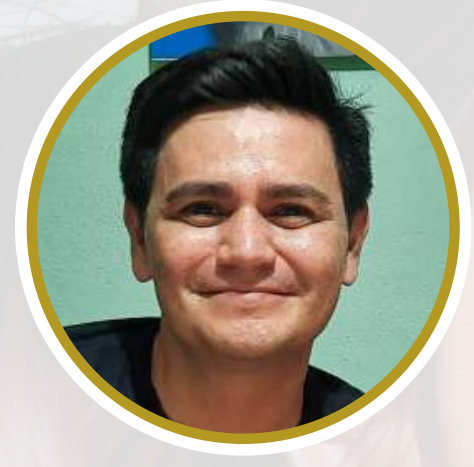

\section{Carlos Enrique Robledo Moreno, 1981, Monterrey, Nuevo León}

Artista egresado de la Facultad de Artes Visuales de la Universidad Autónoma de Nuevo León. La mayor parte de su obra la realiza en murales por las principales avenidas del municipio de San Nicolás de los Garza, Nuevo León, México. Coordina el programa de Arte Urbano en el Instituto Municipal para el Desarrollo Cultural (IMDEC) de San Nicolás de los Garza. Además, realiza su obra en lienzo y en el arte Madonnari (gis/pavimento). Ha expuesto y pintado de manera local, nacional e internacionalmente en paises como Francia, Italia y Estados Unidos. Ha recibido importantes reconocimientos como en Nocera Superiore, Italia, 2013 (2do. lugar del público) y 2015 (1er lugar del público); y en Wilhelmshaven, Alemania, 2019 (3er Lugar Categoría Libre).

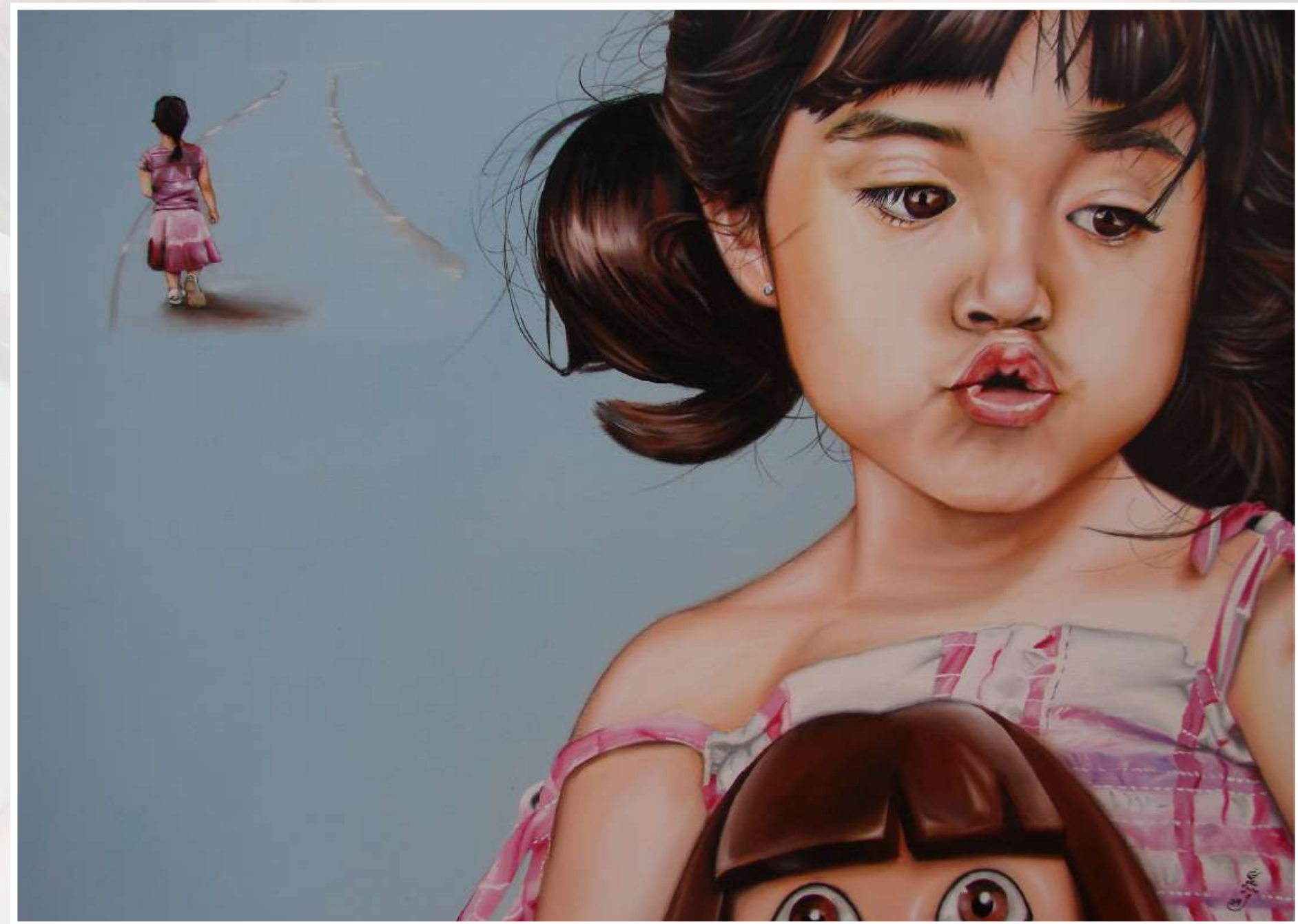

Como artista visual plasmo el retrato y figura humana realista, en conjunto con una simbología acorde a la temática de la obra. Me gusta ver mi obra como todo un proceso, el cual va desde la idea, el boceto, la elección del o la modelo, la toma de una fotografía o bien un dibujo, hasta la realización de la pintura. Generalmente utilizo el acrílico por la rapidez con la que puedo realizar obras en tonos vivos y cálidos. Pienso que en el discurso de la obra que realizo, trato hacer conciencia de lo caótico de la ciudad, del ser humano en sí y de la interacción del individuo con la naturaleza.

\section{- Carlos Robledo}

